\title{
Oscilometría de impulso en adultos: una prueba de función pulmonar complementaria
}

\author{
Impulse oscillometry in adults: a complementary lung \\ function test
}

\author{
Germán DíAz Santos, MD. ${ }^{(1)}$; Gustavo Adolfo HincapiÉ, MD. ${ }^{(2)}$; Jorge Ordóñez, MD. ${ }^{(3)}$; \\ CARLOS AWAD, MD. ${ }^{(4)}$
}

\section{Resumen}

La oscilometría de impulso es una prueba no invasiva que se ha retomado recientemente para determinar la impedancia del sistema respiratorio, principalmente en aquellos pacientes que tengan compromiso de la vía aérea pequeña y cuando no se pueda realizar un esfuerzo adecuado o maniobras convencionales. Puede servir como un método complementario o alternativo a la espirometría en la evaluación del estado del sistema respiratorio. Fue desarrollada desde 1956 pero solo hasta 2007 se incluyó en las guías ATS/ERS para la evaluación de la función pulmonar en preescolares.

Consiste en la medición del impedimento natural al flujo de aire, lo cual incluye pérdidas friccionales y cargas elásticas e inerciales. Mide las resistencias centrales (R20) y las resistencias totales (R5), así como el delta de resistencia ( $\Delta \mathrm{R}=\mathrm{R} 5-\mathrm{R} 20)$, la reactancia capacitiva distal (X5), la frecuencia de resonancia (Fres) y el área de reactancia (AX). Tiene unos criterios de aceptabilidad que deben cumplirse y se sugiere que la interpretación se realice de manera sistemática. Los valores comparativos para su interpretación se han tomado de diferentes ecuaciones de acuerdo con edad, sexo, raza, altura y peso.

Se ha utilizado para evaluar de forma temprana la impedancia del sistema respiratorio en asma y enfermedad pulmonar obstructiva crónica, enfermedades pulmonares intersticiales, cambios pulmonares mecánicos en pacientes con insuficiencia cardíaca congestiva, obstrucción de la vía aérea superior, disfunción de cuerdas vocales, como alternativa para la realización de test de broncoprovocación, como herramienta de evaluación complementaria en el manejo de pacientes con enfermedad pulmonar obstructiva crónica, respuesta a broncodilatadores, cambios en la resistencia de la vía aérea en pacientes con síndrome de apnea/hipopnea obstructiva del sueño, resistencias respiratorias en pacientes con lesión en la médula espinal, entre otras aplicaciones.

Sin embargo, se requieren más estudios de calidad apropiada para determinar la utilidad de esta prueba diagnóstica en la práctica clínica.

Palabras clave: oscilometría de impulso, prueba de función pulmonar.

\begin{abstract}
Impulse oscillometry is a non-invasite test that has been recently reintroduced to determine the impedance of the respiratory system, especially in patients with small-airway involvement and whenever an adequate effort or conventional maneuvers cannot be carried out. It can function as a supplementary or alternative to spirometry in the assessment of the condition of the respiratory system. It was developed since 1956, but only in 2007 it was included in the ATS/ERS guidelines for assessment of lung function in preschool children.

It consists of measuring the natural impediment to airflow, which includes losses due to friction as well as elastic and inertial loads. It measures central resistances (R20) and total resistances (R5), as well as the delta of resistance $(\Delta R=R 5-R 20)$, distal capacitive reactance (X5), the frequency of resonance (Fres) and the area of reactance (AX). It has acceptability criteria that must be met, and it is suggested that the interpretation be made systematically. The comparative values for its interpretation have been taken from different equations according to age, sex, race, height and weight.

It has been used for early assessment of respiratory system impedance in asthma and chronic obstructive pulmonary disease, interstitial lung diseases, mechanical pulmonary changes in patients
\end{abstract}

\footnotetext{
${ }^{(1)}$ Medicina Interna, Fellow de Neumología. Hospital Santa Clara. Bogotá, Colombia. ${ }^{(2)}$ Medicina Interna, Neumólogo. Jefe de Neumología, Hospital Militar Central. Bogotá, Colombia.

(3) Medicina Interna, Neumólogo. Hospital Santa Clara. Bogotá, Colombia.

${ }^{(4)}$ Medicina Interna, Neumólogo. Jefe de Laboratorio del Función Pulmonar, Hospital Santa Clara. Bogotá, Colombia. Correspondencia: Gustavo Adolfo Hincapié Díaz. Correo electrónico: gahincapie@ gmail.com

Recibido: 15/01/14, Aprobado: 20/02/14.
} 
with congestive heart failure, obstruction of the upper airway, vocal chord dysfunction, as an alternative to bronchoprovocation testing in the management of patients with chronic obstructive pulmonary disease, response to bronchodilators, changes in airway resistance in patients with obstructive sleep apnea/hypopnea syndrome, respiratory resistances in patients with spinal injury, among other applications.

However, more studies of adequate quality are required to determine the usefulness of this diagnostic test in clinical practice.

Keywords: impulse oscillometry, pulmonary function tests.

\section{Introducción}

La oscilometría de impulso es una prueba de función pulmonar no invasiva que sirve para el estudio de la mecánica respiratoria, a través de la determinación de la impedancia del sistema respiratorio. Su principal ventaja radica en que no requiere maniobras dependientes del esfuerzo respiratorio del paciente, lo cual elimina la influencia del tono broncomotor en el examen. Está indicada en el estudio del mecanismo fisiopatológico, el diagnóstico y la caracterización de enfermedades pulmonares, principalmente aquellas que tengan compromiso de la vía aérea pequeña, y cuando no se puede realizar un esfuerzo adecuado o maniobras convencionales, situaciones más frecuentes en niños y adultos mayores.

La espirometría es una herramienta de diagnóstico importante y se considera el patrón de oro para tamizar la obstrucción y respuesta broncodilatadora, pero su realización puede ser difícil en pacientes de edad avanzada o en infantes debido a la necesidad de cooperación y requerimiento de maniobras respiratorias específicas. La oscilometría de impulso puede ser una alternativa a la espirometría y da información acerca de las propiedades de resistencia del sistema respiratorio. Esto se evaluó en un estudio que tomó 277 informes de oscilometría de impulso en pacientes entre 65 a 96 años y los comparó con la espirometría, para encontrar que las resistencias medidas con oscilometría de impulso (R5, R5-R20) y frecuencia de resonancia (Fres) se correlacionaron de manera significativa con el $\mathrm{VEF}_{1}$, considerando que la oscilometría de impulso puede servir como un método complementario o alternativo a la espirometría para la evaluación del estado del sistema respiratorio (1). No tiene ninguna contraindicación excepto aquellas alteraciones anatómicas que no permitan realizar una respiración o volumen corriente.
Existen múltiples estudios en niños con resultados favorables para su utilización. Está aceptada en el ámbito internacional como una de las pruebas diagnósticas en el asma y se tiene estandarizada en los consensos en Colombia para el diagnóstico de asma en preescolares (2).

Se hizo una revisión de la literatura para conocer el método de interpretación y evidencia de esta prueba de función pulmonar en adultos.

\section{Historia}

Existen cuatro técnicas para medir las resistencias respiratorias determinando la presión y flujo; éstas son el balón esofágico, la técnica de interrupción, la pletismografía y la técnica de oscilación forzada. En 1956 Dubois y colaboradores describieron la utilidad de las oscilaciones para medir las propiedades mecánicas del pulmón (3). Sin embargo, este método fue olvidado hasta 1965 cuando Muysers retomó estos estudios ya que se habían preferido a la pletismografia como el método ideal para ese momento. En 1971 Smidt y Muysers publicaron por primera vez el método de monofrecuencia oscilatoria para el estudio de las propiedades mecánicas del pulmón.

En 1976 Lander y colaboradores utilizaron la técnica de múltiples frecuencias por medio de señales auditivas pseudovariables en los Estados Unidos, mejorando así muchas de las falencias de la técnica de monofrecuencia. Con el desarrollo de las computadoras esta técnica fue refinándose, primero utilizando modelos animales para determinar efectos broncolíticos y la localización de diferentes efectos fisiológicos en el pulmón (Hantos y col. 1991) (4).

Solo hasta 1981, Müller y Volgel publicaron el escrito "Otro aprovechamiento: oscilometría de impulso", en el que describieron la técnica mejorada por el uso de patrones de ondas rectangulares. Sin 
embargo, fue hasta 1992 que el grupo de trabajo de la Sociedad alemana de neumología, discutió las primeras experiencias sobre este método (4).

Los primeros estudios clínicos descritos fueron hechos por Van Noord en 1990; se realizó diagnóstico diferencial y temprano de enfermedades pulmonares. En 1991 Desager (4) la utilizó para medir la resistencia del tejido a nivel de la nasofaringe.

En los últimos años han aparecido múltiples publicaciones acerca de esta técnica (2, 5). En 2007 las guías ATS/ERS (del inglés American Thoracic Society/European Respiratory Society) la incluyeron como método para la evaluación de la función pulmonar en preescolares (6) y Colombia en sus últimas guías pediátricas del manejo del niño asmático, la recomendó como patrón de oro en el tratamiento del asma en menores de cinco años.

\section{Oscilómetros}

El oscilómetro de impulso es un instrumento que consta de una bocina que genera una presión de pulso $\left(1 \mathrm{~cm}\right.$ de $\mathrm{H}_{2} \mathrm{O}$ ), y produce una oscilación de flujo a determinada frecuencia, que se desplaza a la boquilla donde ingresa a la boca y el árbol respiratorio, siendo contrarios al patrón de flujo espiratorio. Las ondas son reflejadas en el neumotacógrafo calefaccionado y son sensadas en los transductores de presión y flujo que llevan la información a una computadora y transforman las señales análogas a digitales mediante el método de trasformación rápida de Fourier (7).

En la actualidad las casas comerciales venden este equipo con el software que reúne todos los requisitos exigidos por la ATS y la ERS. Requiere una calibración diaria con jeringa de tres litros, previo a su utilización (7).

\section{Principios físicos y fisiopatológicos}

Para entender la oscilometría de impulso se desglosarán algunos principios básicos de la mecánica de la respiración a fin de correlacionarlos con las mediciones en la prueba. Se esquematizan en la figura 1.

\section{Impedancia}

Corresponde al impedimento natural al flujo de aire cuando este intenta desplazarse adentro y afuera del sistema respiratorio. Esta incluye pérdidas friccionales y cargas elásticas e inerciales del sistema, lo que significa que la impedancia es la suma de la resistencia más la reactancia, donde la resistencia equivale a la resistencia total de la vía respiratoria y la reactancia corresponde a la capacitancia, que son los cambios en el volumen periférico dependientes de las propiedades elásticas del pulmón, más la inercia, que es la fuerza que se opone al movimiento de la columna de aire en el árbol bronquial (8).

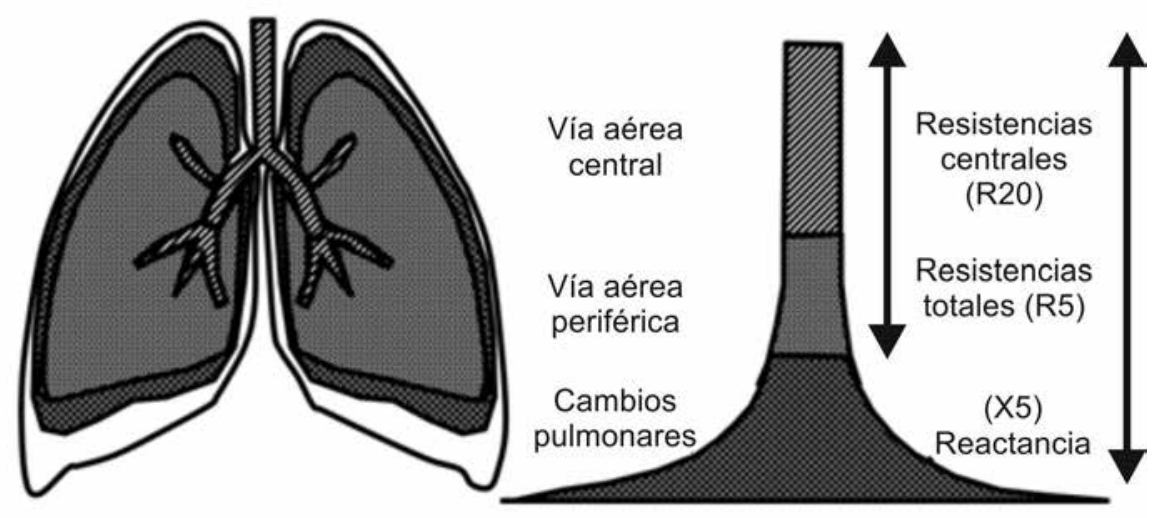

Figura 1. Representación gráfica de los componentes de la oscilometría de impulso y su correlación con sitios anatómicos en el sistema respiratorio. 


\section{Resistencia respiratoria}

Las resistencias respiratorias del pulmón en oscilometría de impulso, están divididas en un componente central y otro periférico. Se denomina R20 a las resistencias centrales y $\mathrm{R} 5$ a las resistencias totales de la vía aérea (suma de las resistencias centrales y periféricas). Cuando sus valores son mayores del 150 por ciento del valor predicho, significa obstrucción. El lugar de la obstrucción depende de la frecuencia de oscilación. En el patrón de obstrucción central o proximal se muestra que el aumento de las resistencias es independiente de la frecuencia de oscilación, mientras que en el patrón de obstrucción distal o periférico se evidencia aumento de las resistencias a bajas frecuencias y disminución de las resistencias a altas frecuencias de oscilación. Con estas diferencias se puede estimar el delta de resistencia $(\Delta \mathrm{R}=\mathrm{R} 5-\mathrm{R} 20)$ que consiste en la diferencia entre las resistencias totales y las resistencias centrales; si son mayores al 20\% del predicho, el patrón de obstrucción es periférico (9).

\section{Reactancia respiratoria}

La reactancia que mide la oscilometría de impulso se denomina reactancia capacitiva distal (X5). Esta evaluación está compuesta por la capacitancia, la cual es de signo negativo y es más prominente a bajas frecuencias de oscilación, y por la inercia que es de signo positivo y predomina en altas frecuencias. También está determinada por la edad del paciente. Si se presenta una disminución mayor a 0,15 del valor predicho se correlaciona con baja capacidad de almacenamiento energético; en este caso se debe correlacionar con una hiperinflación (posiblemente enfisema) o una restricción (tal vez fibrosis) (9).

\section{Frecuencia de resonancia}

La frecuencia de resonancia (Fres) es un punto en el cual la capacitancia y la inercia están en cero a distintas frecuencias. Este valor se eleva tanto en trastornos obstructivos como restrictivos. Su principal utilidad es la evaluación de la respuesta terapéutica y las pruebas de provocación (9).

\section{Área de reactancia}

$\mathrm{El}$ área de reactancia o $\mathrm{AX}$ en la oscilometría de impulso, indica la reactancia respiratoria total de forma cuantitativa. Lo usual es que se mida entre los $5 \mathrm{~Hz}$ y la frecuencia de resonancia. Esta refleja los grados de cambios en la obstrucción de la vía aérea periférica, permitiendo evaluar de forma más sensible la respuesta a los broncodilatadores y también en el seguimiento de los tratamientos a largo plazo (9).

\section{Técnica y criterios de aceptabilidad}

El personal se salud debe explicarle el procedimiento al paciente, quien debe estar sentado con pinzas en la nariz, sosteniéndose las mejillas, sellando la boquilla con los labios y respirando tranquilamente (10).

Debe evitar fumar dos horas antes de la prueba y no usar prendas restrictivas en tórax o abdomen. Si utiliza broncodilatadores debe suspenderlos mínimo cuatro horas previas en el caso de los de corta acción y 12 a 24 horas para los de larga acción. No se debe realizar ejercicio intenso previo a la oscilometría de impulso. Puede ingerir alimentos ligeros ya que no se requiere ayuno (10).

Durante la prueba se realizan como mínimo tres intentos, de los cuales cada uno debe durar como mínimo 30 segundos con cuatro respiraciones en volumen corriente de forma regular. Se deben repetir si hay alguna interrupción o artificio durante o posterior a la realización del intento. Cada intento debe tener un intervalo mínimo de un minuto entre uno y otro intento. Se deben utilizar como mínimo dos frecuencias (las recomendadas $5 \mathrm{~Hz}$ y $20 \mathrm{~Hz}$ ) (10).

Para determinar la reproducibilidad de la prueba se debe tener en cuenta la coherencia de acuerdo con la frecuencia. La coherencia es la relación entre las señales que entran y las que salen. La coherencia a $5 \mathrm{~Hz}$ no debe ser superior a 0,9 y la coherencia a $20 \mathrm{~Hz}$ no debe ser superior a 1,0. La variabilidad entre las mediciones no debe superar el $10 \%$ en las frecuencias superiores a $5 \mathrm{~Hz}$. Para la valoración post-broncodilatador se deben utilizar $400 \mathrm{mcg}$ de broncodilatador de acción rápida (10). 


\section{Cómo interpretarla}

Se sugiere que la interpretación de la oscilometría de impulso al igual que otras pruebas de función pulmonar se realice de manera sistemática, para lo cual se simplifica en cinco pasos sencillos (tabla 1).

\section{Informe y patrones de alteración}

La oscilometría de impulso debe tener los parámetros evaluados en el informe principal (R5, R20, $\Delta \mathrm{R}, \mathrm{X} 5$, Fres, $\mathrm{AX}$ ) junto con las gráficas más importantes de estos para que su interpretación sea fácil y sencilla.

Esta interpretación debe tener unos valores comparables para conocer los valores predichos e interpretar la prueba en un contexto adecuado. Esto ha llevado a múltiples estudios para conocer los valores normales de las resistencias pulmonares (11-15).

Además, se han realizado diferentes ecuaciones teniendo en cuenta edad, sexo, raza, altura y peso. El último estudio disponible para evaluar los valores de referencia se realizó en Alemania en 397 pacientes que nunca habían fumado, con edades entre 45 a 91 años, donde se encontró una variación significativa en las ecuaciones de 34 y $76 \%$ (16). No existen estudios de valores normales de la oscilometría de impulso en pacientes latinoamericanos.

A continuación se resumen los diferentes patrones que se pueden encontrar en la oscilometría de impulso desde el punto de vista gráfico. Se simularon las alteraciones numéricas y se adicionó su correspondiente informe en la tabla 2.

\section{Evidencia del uso de oscilometría de impulso}

Como se anotó, la oscilometría de impulso es una prueba que se ha retomado recientemente. Los estudios en algunas patologías son escasos. Sin embargo el grado de correlación con otras pruebas en general ha sido prometedor. Se hizo una búsqueda de la mejor evidencia científica en algunos escenarios donde la oscilometría de impulso podría ser una prueba complementaria.

\section{Tamización}

La oscilometría de impulso se ha utilizado en la evaluación del asma y de la enfermedad pulmonar obstructiva crónica (EPOC). Un estudio incluyó 95 pacientes con EPOC estable, 52 asmáticos y 29 sanos. En asma se observó un aumento significativo en la resistencia respiratoria a $20 \mathrm{~Hz}$ en comparación con los no fumadores sanos y con la EPOC. Los aumentos en R5 y mayor negativización en X5 fueron notables a medida que aumentó la gravedad de la EPOC. En los pacientes con asma también se observaron aumento en R5 y mayor negativización en $\mathrm{X} 5$ en aquellos con relación $\mathrm{VEF}_{1} / \mathrm{CVF}$ menor a $70 \%$. Se concluyó que la oscilometría de impulso puede ser útil para detectar cambios fisiopatológicos de las vías respiratorias de acuerdo con la gravedad en EPOC e incluso en los asmáticos con $\mathrm{VEF}_{1} / \mathrm{CVF}$ normal (17).

Tabla 1. Pasos para la interpretación sistemática de la oscilometría de impulso.

\begin{tabular}{|c|c|}
\hline Pasos & Característica \\
\hline 1 & - Correlacione las curvas. \\
\hline 2 & - Examine R5 (resistencia total). Es normal o elevada. Si la respuesta es no, continúe a reactancia. \\
\hline \multirow[t]{3}{*}{3} & - Examine R20 (normal o elevada). \\
\hline & - $\mathrm{R} 5-\mathrm{R} 20=>20 \%=$ compromiso de vía aérea periférica \\
\hline & - $\quad \uparrow R 5$ y $\uparrow R 20=$ Central. \\
\hline \multirow[t]{2}{*}{4} & - Examine parámetros de reactancia (X5 y Fres). \\
\hline & - Normal $=X 5 \pm 0,15$. Si es más negativo que este límite, es anormal \\
\hline 5 & $\begin{array}{l}\text { - Fres, es anormal si es mayor que el predicho o disminuyó más del } 20 \% \text { post-broncodilatador (depende de } \\
\text { la indicación del test) }\end{array}$ \\
\hline
\end{tabular}


También puede utilizarse en la detección de enfermedades de forma temprana como en el estudio de Frantz y colaboradores, en el cual participaron 450 pacientes con bronquitis o enfisema clasificados según el GOLD 2012 (Global Initiative for Chronic Obstructive Lung Disease), los cuales se sometieron a oscilometría de impulso, espirometría y un cuestionario de signos y síntomas de la EPOC. Los pacientes sintomáticos tenían mayor resistencia y reactancia pulmonar que los asintomáticos. De igual forma, si los pacientes tenían un GOLD más grave presentaban más alteración en la resistencia y la reactancia. Cuando se compararon los pacientes sintomáticos y que no cumplían los criterios del GOLD, contra quienes cumplían los criterios GOLD y eran sintomáticos, se encontró aumento de las resistencias y reactancias similar en ambos grupos de pacientes, lo que sugiere que la oscilometría de impulso podría detectar alteraciones tempranas (18).

Así mismo, se ha utilizado la oscilometría de impulso para evaluar la capacidad de detectar la gravedad del paciente con EPOC en la cohorte prospectiva ECLIPSE, en la cual se compararon fumadores ( $\mathrm{n}$ $=2054)$, no fumadores $(\mathrm{n}=322)$ y sujetos sanos $(\mathrm{n}$ $=233$ ), y se evaluó si la oscilometría de impulso se correlacionaba con el grado de enfisema en la tomografía. En el grupo de EPOC, la impedancia del sistema respiratorio estaba aumentada en comparación con los controles, pero los intervalos de confianza

Tabla 2. Ejemplos de los diferentes patrones en la oscilometría de impulso.

\section{Patrón normal}
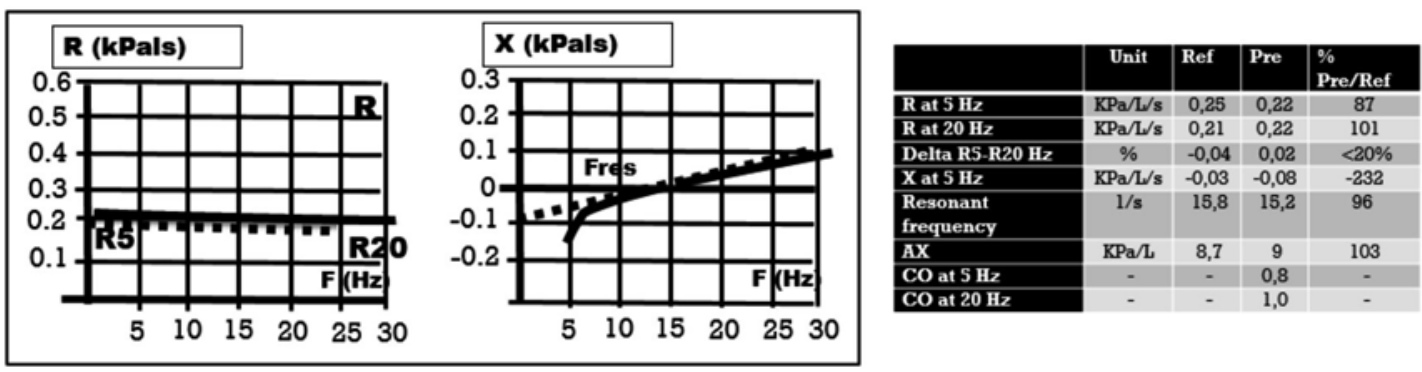

LECTURA: resistencias normales tanto proximal como distal, reactancia capacitiva normal, frecuencia de resonancia dentro de límites normales, área de reactancia adecuada, oscilometría de impulso normal.

Patrón de obstrucción periférica
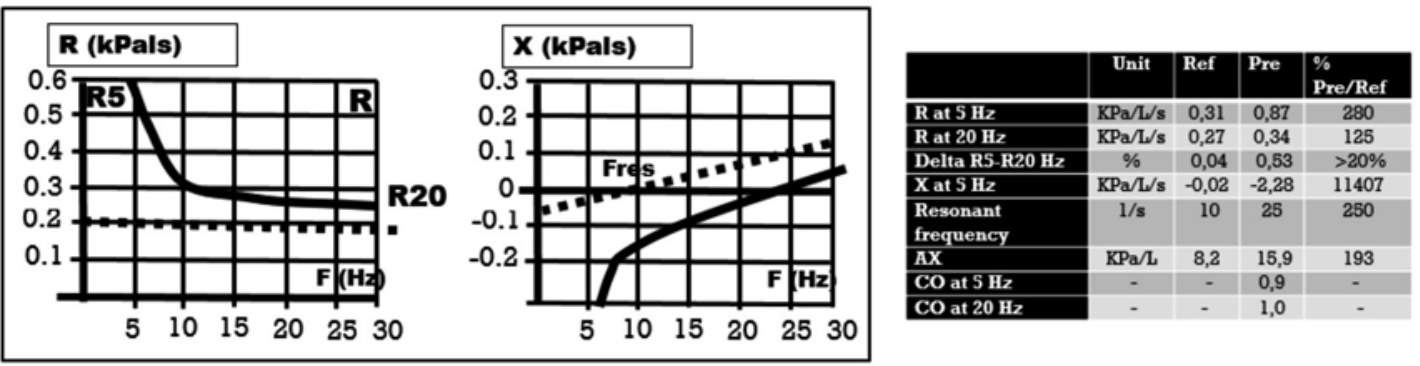

LECTURA: resistencias totales aumentadas con predominio distal, reactancia capacitiva disminuida, frecuencia de resonancia incrementada, área de reactancia aumentada, oscilometría de impulso con patrón de obstrucción periférica. 
Continuación de tabla 2.

\section{Patrón de obstrucción central y periférica}
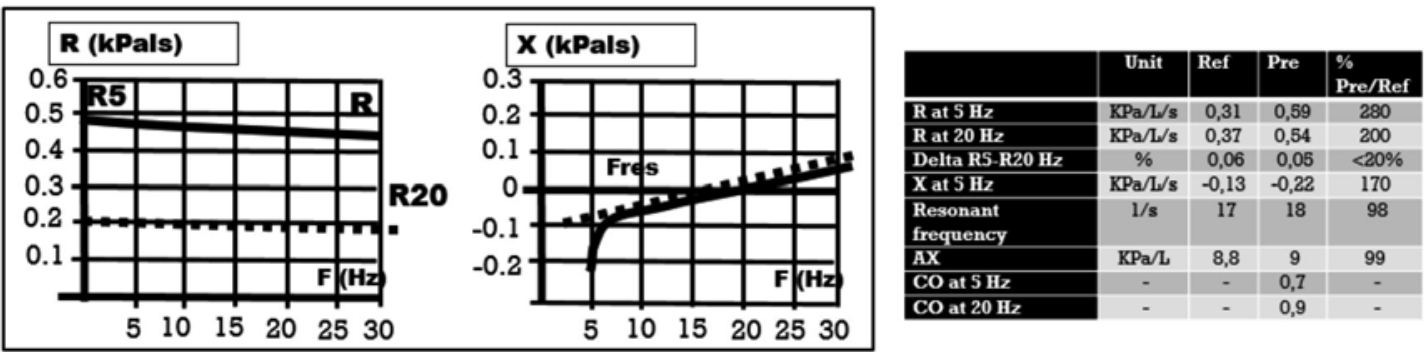

LECTURA: resistencias aumentadas tanto proximal como distal, reactancia capacitiva normal, frecuencia de resonancia dentro de límites normales, área de reactancia normal, oscilometría de impulso con patrón de obstrucción central.

\section{Patrón de restricción}
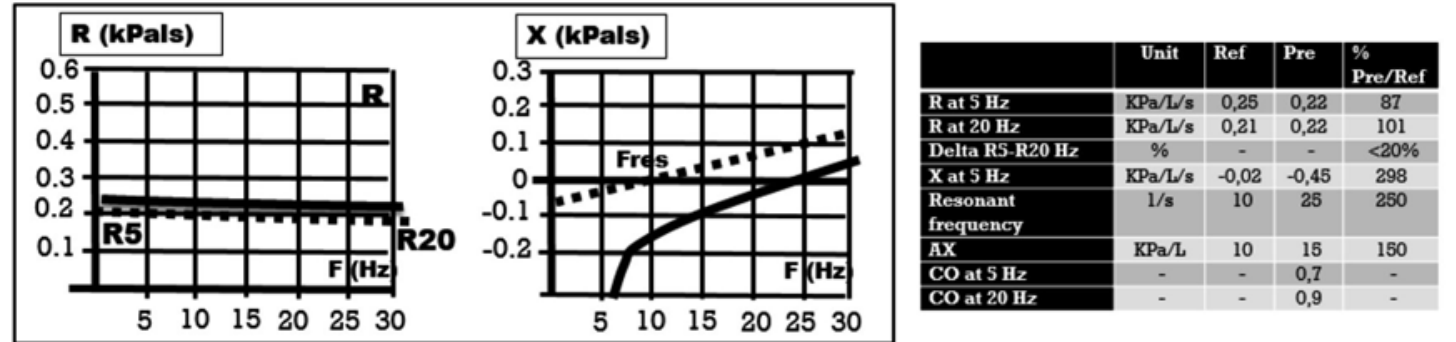

LECTURA: resistencias normales tanto proximal como distal, reactancia capacitiva anormal, frecuencia de resonancia aumentada, área de reactancia aumentada, oscilometría de impulso con patrón de restricción.

\section{Patrón de obstrucción extra torácico}
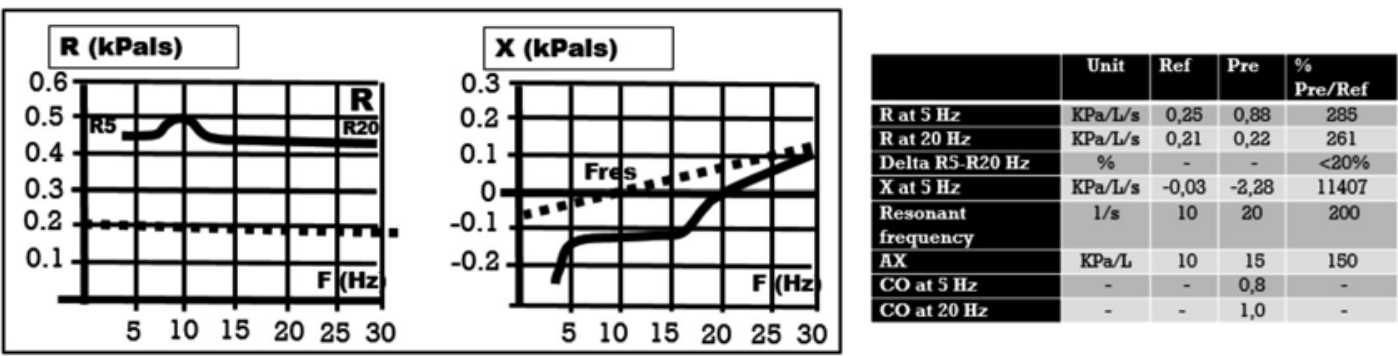

LECTURA: resistencias totales aumentadas a $10 \mathrm{hz}$, reactancia capacitiva disminuida con meseta, frecuencia de resonancia incrementada, área de reactancia aumentada, oscilometría de impulso con patrón de obstrucción extra torácico.

Tomado y modificado de sitio web de Carefusion. (16). 
no fueron satisfactorios. Los valores de los parámetros de impedancia y los índices de tomografía empeoraron con la gravedad GOLD; las correlaciones entre ellos eran pobres. Los datos sugieren que la oscilometría de impulso puede tener una utilidad limitada en la evaluación del grado de enfermedad, y en la actualidad su papel en la evaluación de la progresión de la EPOC permanece indefinido (19).

La oscilometría de impulso puede tener alguna utilidad en los trasplantes pulmonares. En una serie de casos de 22 receptores de aloinjertos pulmonares bilaterales, quienes presentaron cinco rechazos agudos y siete bronquiolitis obliterantes, nueve de ellos tuvieron anormalidades en R5, diez aumentaron en el R5-R10 y doce tuvieron alteraciones de Fres en el área de reactancia, hecho que sugiere que podría ayudar a detectar cambios en los pacientes con trasplante pulmonar (20).

\section{Enfermedad intersticial pulmonar}

La utilidad de la oscilometría de impulso en pacientes con enfermedad pulmonar intersticial ha sido evaluada en un estudio que incluyó 64 pacientes con enfermedad pulmonar intersticial, 54 pacientes con asma, 49 con EPOC y 29 controles. Se demostró un incremento en la resistencia a $5 \mathrm{~Hz}$ en pacientes con enfermedad pulmonar intersticial y disminución de la reactancia a $5 \mathrm{~Hz}$ en comparación con los controles, aunque también se hallaron estas características en pacientes con asma y EPOC. Análisis de los parámetros de la oscilometría de impulso en la fase inspiratoria y espiratoria, demostraron que los cambios en X5 y el área de reactancia $(\mathrm{AX})$ entre la inspiración y la espiración ( $\triangle \mathrm{X} 5$ y $\triangle \mathrm{AX}$, respectivamente) fueron significativamente diferentes en los pacientes con asma, en los pacientes con EPOC y en los controles. Sin embargo, el análisis de regresión lineal múltiple mostró que la presencia de enfermedad pulmonar intersticial se asoció de forma independiente con $\Delta \mathrm{X} 5$, pero no con $\Delta \mathrm{AX}$. Además, $\Delta \mathrm{X} 5$ se correlacionó inversamente con la capacidad vital y la capacidad de difusión del monóxido de carbono en pacientes con enfermedad pulmonar intersticial, lo cual sugiere que $\triangle X 5$ es un rasgo característico en la oscilometría de impulso en pacientes con enfermedad pulmonar intersticial. Este cambio en X5 en la enfermedad pulmonar intersticial podría estar asociado con la severidad y la anormalidad fisiológica; sin embargo, se requieren más estudios (21).

\section{Falla cardíaca}

Se realizó una medición de los cambios pulmonares mecánicos en pacientes con insuficiencia cardíaca congestiva utilizando oscilometría de impulso. Se evaluaron 24 pacientes con falla cardíaca descompensada y 24 controles, mediante la utilización de espirometría y oscilometría de impulso. X5 fue menor y R5 fue mayor en los pacientes con falla cardíaca descompensada que en los controles. Se concluyó así que la oscilometría de impulso puede medir de forma fiable la resistencia de la vía aérea periférica en pacientes con falla cardíaca descompensada y de forma más cómoda que con la espirometría (22).

\section{Obstrucción mecánica de la vía aérea superior}

La obstrucción de la vía aérea superior es una consulta frecuente y la oscilometría de impulso puede ser una alternativa no invasiva para diagnosticarla. Un estudio de 20 pacientes con obstrucción de la vía aérea a quienes se les realizó espirometría, broncoscopia, prueba de disnea y oscilometría de impulso, encontró que a pesar de que la broncoscopia se usa para evaluar pacientes con obstrucción de la vía aérea central, no siempre es posible localizar el sitio anatómico de la obstrucción. La oscilometría de impulso puede discriminar variables de obstrucción de la vía aérea central fija (índice de colapsabilidad menor al 50\%) y además se correlacionó con el índice de disnea (23).

Una serie de casos publicada en 2013, mostró que la oscilometría de impulso puede diagnosticar la disfunción de cuerdas vocales de manera similar a la laringoscopia. La espirometría solo pudo detectar un paciente en esta serie (24).

De otra parte, en un estudio se incluyeron 51 pacientes con sospecha o con asma bronquial comprobada en quienes se hizo visualización directa de las cuerdas vocales por broncoscopia flexible, espirometría y oscilometría de impulso. 
La disfunción de cuerdas vocales fue un hallazgo frecuente en los pacientes con síntomas sugestivos de asma (75\%) y con frecuencia coexiste con asma $(67,65 \%)$. Concluyeron que la oscilometría de impulso es una prueba útil para la detección de la disfunción de cuerdas vocales y fue más sensible que la espirometría (25).

\section{Test de broncoprovocación}

La oscilometría de impulso puede utilizarse como test de broncoprovocación con buena respuesta. Esto fue demostrado en un estudio de 22 pacientes expuestos un alérgeno y ocho a metacolina. Todos tenían diagnóstico de asma alérgica moderada. Se les aplicaron diferentes pruebas de función pulmonar como oscilometría de impulso (R5, R20, R5-R20, X5, AX, Fres), pletismografía (sRaw, sGaw, FRC, volúmenes pulmonares) y espirometría $\left(\mathrm{VEF}_{1}, \mathrm{CVF}, \mathrm{PEF}, \mathrm{FEF} 50 \%\right)$. El índice de oscilometría de impulso más sensible fue R5-R20 y las mediciones de pletismografía y espirometría más sensibles fueron sRaw, sGaw y FEF 50\% con el alérgeno. Después de metacolina, el índice de oscilometría de impulso más sensible fue AX, y la medición de pletismografía más sensible fue sRaw. En general, la oscilometría de impulso (R5$\mathrm{R} 20, \mathrm{AX}, \mathrm{X} 5 \mathrm{~Hz}$ ) demostró ser más sensible que las mediciones de pletismografía y espirometría en la broncoconstricción inducida por alérgenos e inducida por metacolina y puede ser una alternativa cuando la pletismografía y la espirometría no pueden llevarse a cabo (26).

\section{Evaluación de tratamientos instaurados}

La oscilometría de impulso puede usarse en la evaluación del tratamiento de la EPOC. Un estudio reciente evaluó la eficacia del propionato de fluticasona-salmeterol con tiotropio $(n=25)$ versus tiotropio como monoterapia $(n=31)$ en pacientes con EPOC. El grupo fluticasona-salmeterol con tiotropio mostró una mejoría significativa en la resistencia inspiratoria en $5 \mathrm{~Hz}$ y la frecuencia de resonancia, así como la CVF y el $\mathrm{VEF}_{1}$, después de un tratamiento de 12 semanas. No se encontraron diferencias significativas entre las mediciones de oscilometría de impulso y CVF o $\mathrm{VEF}_{1}$, lo cual sugiere que la oscilometría de impulso puede ser una herramienta de evaluación complementaria en el manejo de pacientes con EPOC (27).

Así mismo, se ha utilizado para evaluar la respuesta a los broncodilatadores. En un estudio aleatorizado, transversal con placebo controlado, realizado por Short y colaboradores, se incluyeron 13 pacientes con asma estable leve a moderada persistente, quienes recibieron 10 o $20 \mathrm{mg}$ de propanolol oral, seguidos de histamina, y salbutamol nebulizado $(5 \mathrm{mg})$ para la recuperación. Se realizó espirometría y oscilometría de impulso antes y dos horas después del beta-bloqueador, posterior a histamina y 20 minutos después de salbutamol. Todos los índices de la oscilometría de impulso mostraron un empeoramiento significativo de la resistencia de las vías respiratorias y la reactancia en respuesta al propanolol. En cuanto a la espirometría se presentaron cambios en el $\mathrm{VEF}_{1}$, pero no FEF 25-75. En la broncodilatación, tanto la espirometría como la oscilometría de impulso presentaron cambios, pero fueron más predominantes en la oscilometría de impulso. La magnitud del cambio siempre fue mayor para los parámetros de la oscilometría de impulso, con mayor cambio en AX y R5-R20 en la broncocostricción y la broncodilatación. Finalmente concluyeron que la oscilometría de impulso responde de manera más sensible que la espirometría respecto a la broncoconstricción con propanolol y a la broncodilatación con salbutamol en pacientes con asma moderada (28).

Otro estudio en el que se incluyeron 30 pacientes con diagnóstico de asma leve persistente para evaluar la respuesta a dos combinaciones de corticoides inhalados/agonistas beta-2 de larga acción (propionato de fluticasona/salmeterol 115/21 y budesonida/formoterol 160/4.5), comparó la aparición y extensión de los efectos en las vías respiratorias periféricas después de la primera dosis y posterior a 4 semanas utilizando la oscilometría de impulso. Las resistencias de las vías aéreas disminuyeron y la reactancia mejoró con la dosificación individual de combinaciones de corticoides inhalados/agonistas beta-2 de larga acción. En el asma leve persistente mejoró la función de la pequeña vía aérea y el efecto se mantuvo durante un intervalo de 12 horas por el uso regular durante 4 semanas (29). 


\section{Apnea obstructiva del sueño}

En este sentido también ha sido objeto de estudio la oscilometría de impulso, principalmente por los cambios en las resistencias de las vías aéreas superiores. Como lo demuestran Cao y colaboradores, quienes incluyeron 20 pacientes con síndrome de apnea/hipopnea obstructiva del sueño (SAHOS) severo, 14 pacientes con SAHOS moderado y 10 (controles) sin SAHOS por polisomnografía. Se usó oscilometría de impulso para medir la resistencia de la vía aérea; además, se realizó espirometría en la posición sentada y luego en la supina mientras estaban despiertos. En comparación con los controles, los pacientes con SAHOS tenían cambios significativamente mayores en la impedancia, R5, R20 y R35 debido a cambios en la postura. Se demostró así que los cambios en la resistencia de la vía aérea fueron mayores en las personas con SAHOS, que en quienes no tenían SAHOS (30).

Este hecho fue corroborado por un estudio en 36 pacientes con SAHOS, 14 pacientes con EPOC y 12 pacientes sanos comprobado por polisomnografía. El objetivo fue evaluar la impedancia respiratoria utilizando oscilometría de impulso. Los resultados mostraron que R20 en el grupo de SAHOS fue significativamente mayor que en el grupo EPOC y el control. R5-R20 en el grupo de SAHOS fue menor que en el grupo EPOC, pero significativamente más alto que en el grupo control. Los niveles de R20 y R5-R20 se correlacionaron positivamente con el grado de severidad de la enfermedad. Además, el índice de apnea-hipopnea (AHI) se correlacionó positivamente con R5 y R20. La saturación más baja se correlacionó negativamente con R5 y R5-R20. Se concluyó que la oscilometría de impulso podría utilizarse como una herramienta valiosa para evaluar el grado de obstrucción de la vía aérea superior en los pacientes con SAHOS y que podría ayudar a explorar su mecanismo patológico (31).

\section{Lesión de la médula espinal}

La oscilometría de impulso permite evaluar la dinámica de las vías respiratorias en personas con lesión de la médula espinal porque el esfuerzo del paciente es mínimo, pero la reproducibilidad de medición en lesión de la médula espinal es desconocida.
Para esta razón se evaluaron 10 sujetos con tetraplejía, 10 con paraplejía y 11 individuos sanos. Se obtuvieron oscilometrías de impulso en la posición sentada y supina, y se realizó una evaluación inicial y se repitió el estudio al día siguiente. Se observó que la oscilometría de impulso tiene aplicabilidad en el estudio de la resistencia respiratoria en pacientes con lesión en la médula espinal. Sin embargo, se requieren estudios a largo plazo en pacientes con tetraplejía y paraplejía (32).

\section{Evaluación preoperatoria}

Las pruebas de función pulmonar son útiles para evaluar la condición pulmonar preoperatoria y para detectar un alto riesgo de complicaciones pulmonares postoperatorias. Sin embargo, el esfuerzo espiratorio máximo debe determinarse por espirometría y curva flujo-volumen. Cuando los pacientes no están en capacidad de realizar las maniobras, se obtiene información incorrecta respecto a su sistema respiratorio. Se efectuó un estudio con el objetivo examinar los valores estándar y los efectos sobre la edad de la oscilometría de impulso en pacientes con valoración prequirúrgica. Se analizaron 420 pacientes entre los 20 y 89 años, con función pulmonar normal (\% VCF $>80 \%$ o $\% \mathrm{VEF}_{1}>70 \%$ ), programados para una cirugía electiva. No hubo ningún cambio estadístico en R20-R20 y R5 en los sujetos entre 20 y 60 años, pero se evidenciaron cambios estadísticamente significativos entre el grupo de 70 y 80 años. Los cambios debidos al envejecimiento fueron más fuertes en el flujo espiratorio máximo en $25 \%$ de la capacidad vital forzada (V25), dividido por la altura (V25/Ht), V25/ V50, capacidad vital/capacidad residual funcional y volumen de cierre/capacidad vital que la de oscilometría de impulso. Este estudio muestra que R5-R20 en oscilometría de impulso muestra las condiciones de las vías respiratorias pequeñas sin requerir esfuerzo espiratorio máximo por los pacientes (33).

\section{Enfermedad profesional}

La oscilometría de impulso se ha empleado en la valoración de la polución y en los cambios en el ambiente. Se estudiaron las anomalías respiratorias en los bomberos metropolitanos (488 bomberos de sexo masculino, de los cuales $25 \%$ eran 
exfumadores y $10 \%$ eran fumadores actuales) con exámenes respiratorios de rutina por espirometría y evaluación de los síntomas respiratorios normales. Para ello se usó la espirometría, la oscilometría de impulso y un cuestionario de síntomas. La espirometría detectó anomalías en el 12\%, la oscilometría de impulso fue anormal en un $9 \%$ y los sujetos con síntomas respiratorios correspondieron al 20\%. El tabaquismo actual se asoció con elevación todos los parámetros de la oscilometría de impulso y con FEF, tos productiva crónica y disnea. La exposición durante tareas de extinción de incendios se asocia con tos productiva crónica, pero no fue significativa con la espirometría o la oscilometría de impulso. El uso de oscilometría de impulso para la evaluación de la salud respiratoria en los bomberos, identificó disfunción de las vías respiratorias en algunos individuos, incluso cuando los valores de espirometría estaban dentro del rango normal y no había síntomas respiratorios (34).

\section{Conclusiones}

La oscilometría de impulso es un estudio complementario a las pruebas de función pulmonar actuales y está indicada para evaluar pacientes adultos que no puedan realizar maniobras de los exámenes convencionales, para la detección de alteraciones obstructivas (centrales y periféricas) y restrictivas y para el ajuste de tratamientos. Es fácil de aplicar e interpretar si se emplea una técnica adecuada. No obstante, se requieren más estudios, con muestras más representativas, con el objetivo de establecer las diferentes utilidades y beneficios de este examen en la práctica clínica.

\section{Bibliografía}

1. Tomalak W1, Czajkowska-Malinowska M, Radliński J. Application of impulse oscillometry in respiratory system evaluation in elderly patients. Pneumonol Alergol Pol. 2014;82(4):330-5. DOI: 10.5603/PiAP.2014.0041.

2. Dencker M, Malmberg LP, Valind S, Thorsson O, Karlsson $\mathrm{MK}$, et al. Reference values for respiratory system impedance by using impulse oscillometry in children aged $2-11$ years. Clin Physiol Funct Imaging. 2006;26:247-50. DOI: 10.1111/j.1475097x.2006.00682.

3. Dubois AB, Brody AW, Lewis DH, Burgess BF. Oscillation mechanics of lung and chest in man. J Appl Physiol. 1956;8:587-94.
4. Vogel J, Smidt U. Impulse oscillometry. Analysis of lung mechanics in general practice and the clinic, epidemiological and experimental research. Frankfurt am Main, Alemania: Ed. PMI Verlagsgruppe $\mathrm{GmbH} ; 1994$.

5. Cavalcanti JV, Lopes AJ, Jansen JM, Melo PL. Detection of changes in respiratory mechanics due to increasing degrees of airway obstruction in asthma by forced oscillation technique. Resp Med. 2006;100(12):2207-19. DOI: 10.1016/j. rmed.2006.03.009

6. Beydon N, Davis SD, Lombardi E, Allen JL, Arets HG, Aurora P. An official American Thoracic Society/European Respiratory Society statement: pulmonary function testing in preschool children. Am J Respir Crit Care Med. 2007;175:1304-45.

7. Smith HJ, Reinhold P, Goldman MD. Forced oscillation technique and impulse oscillometry. Eur Respir Mon. 2005;31:72105.

8. Kaczka DW, Dellacá RL. Oscillation mechanics of the respiratory system: applications to lung disease. Critical Reviews ${ }^{\mathrm{TM}}$ in Biomedical Engineering. 2011; 39(4):337-59.

9. Al-Mutairi SS, Sharma PN, Al-Alawi A, Al-Deen JS. Impulse oscillometry: an alternative modality to the conventional pulmonary function test to categorize obstructive pulmonary disorders. Clin Exp Med. 2007;7(2):56-64.

10. Smith HJ, Reinhold P, Goldman MD. Forced oscillation technique and impulse oscillometry. Eur Respir Mon. 2005;31:72105.

11. Oostveen E. The forced oscillation technique in clinical practice: methodology, recommendations and future developments. Eur Respir J. 2003; 22:1026-41.

12. Guo YF, Herrmann F, Michel JP, Janssens JP. Normal values for respiratory resistance using forced oscillation in subjects $>65$ years old. Eur Respir J. 2005; 26:602-8.

13. Shiota S, Katoh M, Fujii M, Aoki S, Matsuoka R, Fukuchi Y. Predictive equations and the reliability of the impulse oscillatory system in Japanese adult subjects. Respirology. 2005;10:310-5.

14. Smolej Narancić N, Pavlović M, Zuskin E, Milicić J, SkarićJurić T, Barbalić M, Rudan P. New reference equations for forced spirometry in elderly persons. Respir Med. 2009;103: 6218. DOI: $10.1016 /$ j.rmed.2008.10.013

15. Schulz H, Flexeder C, Behr J, Heier M, Holle R, Huber RM, et al. KORA Study Group. Reference values of impulse oscillometric lung function indices in adults of advanced age. PLoS One. 2013;8(5):e63366. DOI: 10.1371/journal.pone.0063366. Print 2013.

16. Carefusion; IOS impulse spirometry, Spirometric and airway resistance diagnostics combined in one compact device. IOS Interpretation poster. Disponible en: : http://www.carefusion. com/pdf/Respiratory/Pulmonary_Function_Testing/IOS_Poster_Typical_Curves.pdf; (Acceso 27 Sep 2014).

17. Kanda S, Fujimoto K, Komatsu Y, Yasuo M, Hanaoka M, Kubo $\mathrm{K}$. Evaluation of respiratory impedance in asthma and COPD by an impulse oscillation system. Intern Med. 2010;49(1):2330. Epub 2010 Jan 1. 
18. Frantz S, Nihlén U, Dencker M, Engström G, Löfdahl CG, Wollmer P. Impulse oscillometry may be of value in detecting early manifestations of COPD. Respir Med. 2012;106(8):111623. DOI: 10.1016/j.rmed.2012.04.010.

19. Crim C, Celli B, Edwards LD, Wouters E, Coxson HO, Tal-Singer R, et al. ECLIPSE investigators. Respiratory system impedance with impulse oscillometry in healthy and COPD subjects: ECLIPSE baseline results. Respir Med. 2011;105(7):1069-78. DOI: 10.1016/j.rmed.2011.01.010.

20. Goldman MD, Saadeh C, Ross D. Clinical applications of forced oscillation to assess peripheral airway function. Respir Physiol Neurobiol. 2005; 148:179-94.

21. Sugiyama A, Hattori N, Haruta Y, Nakamura I, Nakagawa $\mathrm{M}$, Miyamoto $\mathrm{S}$, et al. Characteristics of inspiratory and expiratory reactance in interstitial lung disease. Respir Med. 2013;107(6):875-82. DOI: 10.1016/j.rmed.2013.03.005.

22. Nourizadeh M, Ghelich Y, Amin A, Eidani E, Gholampoor Y, Asadmoghadam M, et al. Study the mechanical pulmonary changes in patients with congestive heart failure (CHF) by impulse oscillometry. J Cardiovasc Dis Res. 2013;4(2):130-3. DOI: 10.1016/j.jcdr.2012.06.001.

23. Handa H, Huang J, Murgu SD, Mineshita M, Kurimoto N, Colt HG, Miyazawa T. Assessment of central airway obstruction using impulse oscillometry before and after interventional bronchoscopy. Respir Care. 2014;59(2):231-40. DOI: 10.4187/ respcare.02094.

24. Komarow HD1, Young M2, Nelson C3, Metcalfe DD3. Vocal cord dysfunction as demonstrated by impulse oscillometry. J Allergy Clin Immunol Pract. 2013;1(4):387-93. DOI: 10.1016/j. jaip.2013.05.005.

25. Hira HS, Singh A. Significance of upper airway influence among patients of vocal cord dysfunction for its diagnosis: Role of impulse oscillometry. Lung India. 2009;26(1):5-8. DOI: 10.4103/0970-2113.45197.

26. Naji N, Keung E, Kane J, Watson RM, Killian KJ, Gauvreau GM. Comparison of changes in lung function measured by plethymography and IOS after bronchoprovocation. Respir Med. 2013;107(4):503-10. DOI: 10.1016/j.rmed.2012.12.022.

27. Mineshita M, Shikama Y, Nakajima H, Nishihira R, Komatsu $\mathrm{S}$, Kubota M, et al. COPD EXHALATION Investigators. The application of impulse oscillation system for the evaluation of treatment effects in patients with COPD. Respir Physiol Neurobiol. 2014;202:1-5. DOI: 10.1016/j.resp.2014.07.008.

28. Short PM, Williamson PA, Lipworth BJ. Sensitivity of impulse oscillometry and spirometry in beta-blocker induced bronchoconstriction and beta-agonist bronchodilatation in asthma. Ann Allergy Asthma Immunol. 2012;109(6):412-5. DOI: 10.1016/j. anai.2012.09.010.

29. Diong B1, Singh K, Menendez R. Effects of two inhaled corticosteroid/long-acting beta-agonist combinations on smallairway dysfunction in mild asthmatics measured by impulse oscillometry. J Asthma Allergy. 2013;6:109-16. DOI: 10.2147/ JAA.S48827.

30. Cao J, Que C, Wang G, He B. Effect of posture on airway resistance in obstructive sleep apnea-hypopnea syndrome by means of impulse oscillation; Respiration. 2009;77(1):38-43. DOI: $10.1159 / 000114146$.

31. Liu H1, Ni W, Zhao J, Xiong S, Xu Y, Zhang Z. The diagnosis value and its implication of impulse oscillometry in obstructive sleep apnea syndrome patients. J Tongji Med Univ. 2000;20(4):280-2.

32. Cirnigliaro CM, Lesser M, Moyer J, Kirshblum SC, Bauman WA, Spungen AM. Reproducibility and effect of posture on impulse oscillation parameters in persons with spinal cord injury. J Spinal Cord Med. 2012;35(1):28-34. DOI: 10.1179/2045772311Y.0000000047.

33. Fujiwara K. Preoperative lung function tests using impulse oscillometry. Masui. 2010;59(2):169-78.

34. Schermer T, Malbon W, Newbury W, Holton C, Smith M, Morgan M, Crockett A. Spirometry and impulse oscillometry (IOS) for detection of respiratory abnormalities in metropolitan firefighters. Respirology. 2010;15(6):975-85. DOI: 10.1111/j.14401843.2010.01809.x. 